$10.2478 / \mathrm{gb}-2020-0004$

sciendo

\title{
„Schwankend zwischen zwei Kulturen“6. Einige Bemerkungen zur deutschlandbezogenen Gelegenheitsdichtung Ion Barbus
}

\section{Ioana CRĂCIUN-FISCHER}

Prof. Dr.; Universität Bukarest; E-Mail: craciunfischer@yahoo.com

\begin{abstract}
The Romanian poet and internationally acclaimed mathematician Ion Barbu (i.e. Dan Barbilian), 1895-1961, practiced in his occasional poetry related to his experience as a doctoral student and later as a visiting professor in interbellic Germany a poetic discourse of immediate, sometimes diary-like reflection. The vitality of his occasional poetry mainly addressed to his close friends and seldom intended for publication is fed by the permanent contrast between the German and the Romanian culture and civilization. The paper analyzes the intercultural dialogue which constitutes the background of Ion Barbu's Germany-related occasional poetry with special emphasis on his poems written in German.
\end{abstract}

Key Words: Ion Barbu, Dan Barbilian, Tudor Vianu, Heinrich Heine, Weimar Republic, protestantism, sexuality, occasional poetry, mathematics and poetry, cross cultural dialogue, National Socialism

Der Dichter Ion Barbu (1895-1961), in der Welt der Höheren Mathematik unter dem Namen Dan Barbilian bekannt, erhielt nach seinem Studienabschluss 1921 in Bukarest ein Promotionsstipendium, mit dem er nach Deutschland reisen konnte. Sein Gedichtband După melci, 1921 in Bukarest mit Illustrationen von $\mathrm{M}$. Teişanu erschienen, ließ eine ungewöhnliche Doppelbegabung erahnen, die Zahlen und Worte, abstrakte Formeln und anschauliche Metaphern, mathematische Präzision und 
poetische Suggestion mit derselben Virtuosität zu orchestrieren fähig war. Drei Jahre verbrachte der rumänische Mathematikdoktorand und Lyriker armenischer Herkunft in Deutschland, in der eher halbherzig verfolgten Absicht, die Doktorwürde zu erwerben. Der Briefwechsel mit seinem damals in Tübingen über das Wertungsproblem in Schillers Poetik promovierenden Freund, dem Literaturhistoriker, Ästhetiker, Kritiker und Übersetzer Tudor Vianu, zeugt von einem hedonistisch geführten, abenteuerlustigen Leben in Göttingen, Hamburg und Berlin, das eher um Wein, Weib und Rauschgift denn um Wissenschaft, Forschung und Promotion kreiste. Es gelang Dan Barbilian alias Ion Barbu erst 1929, fünf Jahre nach seiner Rückkehr nach Rumänien, die Promotion im Fach Mathematik erfolgreich abzuschließen. Unter der Anleitung des berühmten Mathematikprofessors Gheorghe Ţiţeica, dessen Assistent er in Bukarest wurde, verfasste er eine Dissertation mit dem Titel Reprezentarea canonică a adunării funcţiilor ipereliptice, die den Anfang seiner wissenschaftlichen Karriere als eines Algebraikers und Geometrikers von Weltrang markieren sollte. Die Vorträge, die Dan Barbilian alias Ion Barbu 1936 in Hamburg und in Göttingen und zwei Jahre später in Münster als Gastprofessor abhielt, boten dem inzwischen international gefeierten Mathematiker Dan Barbilian und dem in Rumänien nach der Publikation des Gedichtbands Joc secund (1930) zunehmend akklamierten Dichter Ion Barbu die Möglichkeit, den Kontakt zur deutschen Sprache, zur deutschen Kultur und Zeitgeschichte aufrecht zu erhalten bzw. immer wieder aufzufrischen. Maßgeblich trug auch die Cottbuserin Gerda Barbilian, die äußerst devote deutsche Ehefrau des Dichters, dazu bei, Deutschland zum festen Bezugspunkt im Leben Ion Barbus und Deutsch zur zweiten Sprache seiner deutschlandbezogenen Gelegenheitsdichtung werden zu lassen.

Sowohl die "wilden Zwanziger" der Weimarer Demokratie als auch die Jahre der nationalsozialistischen Diktatur fanden 
Eingang in Ion Barbus Gelegenheitsdichtung. Es handelt sich dabei um Texte, die oft einen einzigen Adressaten innerhalb des nahen Freundeskreises des Dichters haben und nicht immer für die Veröffentlichung vorgesehen waren, zumal sie höchst Intimes, nicht selten auch Pikantes und sogar derb Obszönes beinhalteten. Fernab aller Hermetik praktizierte Ion Barbu in seiner deutschlandbezogenen, ab und zu gänzlich auf Deutsch verfassten Gelegenheitslyrik einen poetischen Diskurs der unmittelbaren, zuweilen tagebuchartigen Reflexion, deren Vitalität sich aus der permanenten Kontrastierung zwischen dem Eigenen und dem Fremden speiste und bald im exaltierten Lob des Eigenen, bald in der Bejubelung des Fremden gipfelte. Dieser Lyrik, die als Ergebnis eines intensiv gepflegten interkulturellen Dialogs entstand und dem beständigen Bedürfnis des Dichters nach einer Auslotung der eigenen Identität entsprach, gilt im Folgenden meine Aufmerksamkeit. Methodisch erfolgt dies im Bewusstsein, dass man dabei ein Sprachterritorium betritt, das sich nur bedingt für eine literaturwissenschaftliche Analyse eignet, da die zu untersuchenden Texte in erster Linie Lebensdokumente darstellen.

Die Begegnung mit dem Fremden nimmt in Ion Barbus Gelegenheitsdichtung immer wieder sinnliche Formen an, wobei das erotische Abenteuer oft zum Initiationsritus wird, der das lyrische Ich geistig formt und bereichert. In dem einer Frau aus dem Norden gewidmeten Gedicht mit dem Titel Convertire, das 1924 in der Zeitschrift Cugetul românesc veröffentlicht wurde, wird der Liebesakt mit einer Berliner Madame Bovary, die als Helene Braun, Ehefrau eines deutschen Generals, identifiziert wurde, zu einer Lebenslehre über den Unterschied zwischen einer Dame und einem Weib, d.h. nicht zuletzt über den Unterschied zwischen Natur und Kultur: „Mi-ai lunecat în braţe, ai desfăcut fuior / Tot părul, ca un galben, întins eter der miere... / Şi m-ai făcut cu-un lucru de preţ mai ştiutor: // Că două 


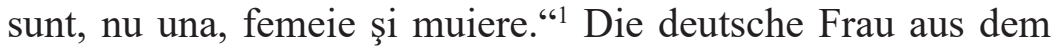
Norden mit ihrem weißen, reinen Fleisch -,,Intâia mea cucoană de voaluri şi mătăsuri“" (S. 171) - fungiert in diesem Gedicht, gleich dem hohen wîp des mittelalterlichen Minnesangs, als Lehrmeisterin des linkischen Jünglings aus der Walachei, als welchen sich das lyrische Ich selbstironisch bezeichnet. Die Varianten des Schlussverses, die in Ion Barbus Gedichtmanuskript zu lesen sind (z. B.: „Din fagure-i femeea, din scroafă e muierea“; „Că din potir, femeea-i. Din scroafă-i muierea“, S. 669), verdeutlichen in ihrer Derbheit die Antithese zwischen Dame und Weib, wobei die Dame als Personifikation Deutschlands und seiner hohen Kultur interpretiert werden kann, während das Weib - oder als derbe sprachliche Variante: die Sau die walachische Heimat in ihrer ursprünglichen Natürlichkeit repräsentiert.

Während die Gestalt der blonden Frau aus dem Norden auf Gustave Flauberts Romanheldin Madame Bovary anspielt und ihre Literarisierung zur Kaschierung ihrer wahren Identität beiträgt, die lediglich in Ion Barbus privater Korrespondenz mit Tudor Vianu enthüllt wird, tritt in Ion Barbus Gedicht $C u$ şapca studenţească... die historisch bezeugte Lily Lang zwar unter ihrem realen Namen, zugleich jedoch auch in literarisierter Gestalt auf: Lily Lang, die blonde Walküre ${ }^{2}$, ist „Romantica din Cassel cu-atâta Sturm-und-Drang!“*3 (S. 219). Auch Lily Lang wird als Kulturträgerin geschildert, die in ihrer Person

${ }^{1}$ Barbu, Ion: Convertire, in: Ders., Opere. I. Versuri. Ediţie alcătuită de Mircea Coloşenco. Bucureşti, 2000, S. 171f. Hier: S. 172. Die Gedichte Ion Barbus werden im fließenden Text mit Angabe der jeweiligen Seitenzahl nach dieser Edition zitiert, die unter der Ägide der Rumänischen Akademie in der von Eugen Simion koordinierten Sammlung Opere fundamentale veröffentlicht wurde.

2 Ion Barbu rumänisiert in diesem Gedicht das deutsche Substantiv Walküre zu ,valkiră““, so wie er im Gedicht Tante Buhr 1783 (S. 263) das Substantiv „Hure“ zu „Hură“ rumänisiert!

3 Kursivschrift im Original! 
einerseits den germanischen Mythos evoziert, so wie man ihn aus Richard Wagners Ring-Tetralogie kennt, andererseits aber auch an Goethe und dessen Verlobte Lili Schönemann denken lässt.

Manchmal fungieren weibliche Gestalten aus der deutschen Literaturgeschichte als Kontrastfolie zu realen Partnerinnen aus dem Leben des Dichters. Heinrich Heines Loreley wird beispielsweise in einem Tudor Vianu gewidmeten Gedicht mit einem realen Kölner Mädchen namens Lo verglichen, mit dem das lyrische Ich dreimal in der Nacht die Messe läutet, worunter es scherzend eine sexuelle Orgie versteht: „Azi noapte: noapte de orgii, / Am sunat mesa de trei ori!! / Iar, azi, plin de mitologii, / Cu Lo de după subţiori, / Gândesc la tine / Şi la Udine..." (Pe Rin..., S. 221). Metaphern dieser Art, die in euphemistischer Manier die gesellschaftlich tabuisierte Sexualität und ihre freie Erfüllung beschwören, kennt man aus der Bildersprache des spätmittelalterlichen Fastnachtspiels. Verkörperte Heinrich Heines Loreley den Typus der femme fatale, so ist Ion Barbus Kölner Lo eher mit dem süßen Mädel des Wiener Volkstheaters geistig verwandt.

Auf Heinrich Heines Lyrik greift Ion Barbu auch im Gedicht Dedicaţie zurück, das er der Dichterin Nina Cassian - „,nefireasca - nespus de albă! - Nina“ (Ut algebra poesis, S. 297) -, seiner fast dreißig Jahre jüngeren Freundin, widmet, deren Anfänge auf literarischem Gebiet mit dem Gedichtband $L a$ scara 1/1 (1947) im Zeichen der rumänischen Avantgarde standen. Das Gedicht Dedicaţie, dessen Titel auf das Prinzip „ut pictura poesis" der Horazschen Poetik anspielt, evoziert einen Besuch Ion Barbus im Geburtshaus Heinrich Heines in der Düsseldorfer Bolkerstraße $\mathrm{e}^{4}$, wo eine Kohlezeichnung zu bewundern war, die den Dichter des Vormärz sitzend zeigte. Nina Cassian war wie Heinrich Heine selbst eine getaufte Jüdin. Die

${ }^{4}$ In Ion Barbus Gedicht wird der Straßenname fälschlicherweise als „Bolkenstrasse“ wiedergegeben! 
ins Gedicht in rumänischer Übersetzung Ion Barbus eingeflochtene Strophe aus Heinrich Heines Gedichtzyklus Die Heimkehr (,Jene Flammen sind erloschen, / Und mein Herz ist kalt und trübe, / Und dies Büchlein ist die Urne / Mit der Asche meiner Liebe. ${ }^{(5)}$ ) lautet wie folgt: „Stinse flăcări. Arsă, struna / S-a lăsat în arcul lirei. / Cartea singură mi-e urna / Cu cenuşile iubirii." " (Dedicaţie, S. 192.) Ion Barbu vergleicht Heinrich Heines melancholisches Lied mit einem ,cânt“, einem Lied, der jüdischen Lyrikerin Nina Cassian und stellt dabei eine Ähnlichkeit zwischen den beiden Werken fest, die er sowohl im Ästhetischen als auch im Ethnischen begründet sieht: „Un cânt de mat argint al palei Nine / Mai greu ca orbul lumei îl socot. / Curând un glas vom fi suind în tine / Cum Heine sună-ntr-însul versul got.“ (Ebd.) Der deutsche Jude Heinrich Heine und die rumänische Jüdin Nina Cassian sind im Gedicht Ion Barbus dank ihrer Wortkunst und ihrer Herkunft Wahlverwandte. Durch seine Liebe zur dichtenden Nina identifiziert sich das lyrische Ich mit dem Schicksal Heinrich Heines, den er als „Evreul Botezat" (ebd.), als getauften Juden, bezeichnet und dessen Pariser Exil ihn an die eigenen in der Fremde verbrachten Jahre erinnert: „Falangele acestei oboseli / De alge dezlegate către sud, / A tâmplei umbră din străine seri: / Cu drumul meu, atunci, le-am mai văzut.“ (Ebd.)

Unter Ion Barbus deutschlandbezogenen Gelegenheitsgedichten gibt es auch Werke, in denen die Frau als unerotisches, unattraktives, ja als abstoßendes Geschöpf geschildert wird. Dies ist insbesondere der Fall, wenn die betreffende Frau der protestantischen Konfession angehört. Das lyrische Ich macht den sinnenfeindlichen Geist der Lehre Martin Luthers dafür verantwortlich, dass Protestantinnen als Liebhaberinnen anscheinend nichts taugen. Ion Barbu zitiert in der Originalsprache den

${ }^{5}$ Heine, Heinrich: Die Heimkehr, LXXXVIII, in: Ders.: Sämtliche Schriften in zwölf Bänden. Band 1. Schriften 1817-1840. Herausgegeben von Klaus Briegleb, Frankfurt am Main, Berlin, Wien, 1981, S. 149.

${ }^{6}$ Kursivschrift im Original! 
ersten Vers des Kirchenlieds Martin Luthers Ein'feste Burg ist unser Gott, bezeichnet die protestantische Bekenntnishymne als unfruchtbar und vergleicht sie mit einem todbringenden Messer in der Wunde des lutherischen Fleisches: „Nu știi, Oriental Magot, / Cum imnul sterp (cuţit în rană) / Distruge carnea luterană!..." (S. 209). ${ }^{7}$ Daher der (scherzhaft gemeinte) Ratschlag an seinen Busenfreund Tudor Vianu, vor der protestantischen Frau wie vor einer damals bekannten Bukarester Prostituierten namens Getta Popa zu fliehen: „Dar fugi... ca de Getta Popa, / Când vezi dama protestantă! ‘" (Bea, S. 220.) Als erotische Alternative zu den seinem Geschmack nach zu mageren deutschen Frauen (,De când îmi port trudiţii paşi / Pe la Reichsdorf şi la Terasă / N-am întâlnit nemţoaică grasă!“ (Ein fester Burg [sic!], S. 209), sehnt sich das lyrische Ich nach den Fettpolstern - ,perne (ebd.) - der Jüdinnen Bukarests und Göttingens, die in typisch antisemitischen Klischees zwar als schmutzig, dank ihrer körperlichen Fülle aber dennoch als sexuell attraktiv beschrieben werden: ,-- Şi fără perne $e^{9}$ ce să faci? / (Hei! ovreicuța din Covaci, / Din Văcăreşti ori indigena / Cartoafă-n ceartă cu igiena!) // Le defăimam, dar mă căiesc;““(ebd.). Die ersehnte Rückkehr nach Bukarest ins jüdische Bordell „la ţaţa Frosa“ (ebd.) wird mit einer der deutschen Geschichte entnommenen Metapher als Gang nach Canossa humorvoll beschrieben (s. ebd.).

${ }^{7}$ Der Titel des Gedichts Ein fester Burg... [sic!] enthält grobe Grammatikfehler, die im ersten Vers sogar vermehrt auftreten: „Ein fester burg ist unser gött" [sic!]. Da der Herausgeber der im vorliegenden Beitrag zitierten Edition - Mircea Coloşenco - in seinem Kommentar diesen Gedichttitel durch „Un oraş în sărbătoare este Dumnezeul nostru“ (sic!, S. 732) grundfalsch übersetzt, ist man berechtigt, anzunehmen, dass er des Deutschen nicht mächtig ist. Es ist daher nicht auszuschließen, dass die von mir monierten Grammatikfehler de facto Transkriptionsfehler darstellen, die bereits in der ersten Veröffentlichung des Gedichts in der Zeitung Adevărul literar şi artistic, IX, Nr. 528 vom 25. Juli 2000, S. 3 begegnen und später nicht stillschweigend korrigiert wurden.

${ }^{8}$ Kursivschrift im Original

9 Kursivschrift im Original 
Für Ion Barbu verkörpert die deutsche Geschichte, insbesondere das germanische Heidentum mit seinen Legenden, Riten und Mythen, den Kulturraum einer orgiastisch sich entfaltenden Sexualität, deren ästhetisches Erlebnis ihm die verführerisch singenden Rheintöchter in Richard Wagners RingTetralogie vermitteln: „Îmi simt un mădular marin / De viking supt de continente / Pe Flut-ul Elbei eminente, / Pe viersul ielelor din Rin..." (St. Paula..., S. 262). Diese überbordende, im Gedicht Tante Buhr 1783 (S. 263) in Bildern der antiken Mythologie thematisierte Sexualität wird im Bordell der berühmt-berüchtigten Hamburger Liebesdienerin zu einem realen Erlebnis, das den aristokratisch lebenden Freund in der Ferne - „O, Tudor drag, o, Duh chiabur" - zur uneingeschränkten Erfahrung der eigenen Sexualität auffordert: „Te cheamă Hamburgul Tantei Buhr..." (ebd.). In ironisierender Absicht schildert der Dichter die sexuellen Orgien im Etablissement der zwielichtigen Zeitgenossin G. E. Lessings, Tante Buhr, als kultische Handlung, als archaischen Ritus, der in mystischer Ekstase gipfelt: „Prompţi coribanţi şi coribante / Se zbat în cinstea Marei Tante." (Tante Buhr 1783, S. 263.)

Dem zügellosen Erlebnis des Eros wohnt in den Gelegenheitsversen Ion Barbus immer ein kultur- oder literarhistorisches Moment inne, das die Trivialisierung der Sexualität zu einer rein fleischlichen Erfahrung verhindert. Enthält das dem Musik liebenden Freund Alexandru Rosetti gewidmete Gedicht St. Paula ... eine Anspielung auf Richard Wagner als den Komponisten der "Ring"-Tetralogie, so wird in dem Tudor Vianu gewidmeten Gedicht Tante Buhr 1783 G.E. Lessing als der Verfasser der Hamburgischen Dramaturgie ${ }^{10}$ namentlich erwähnt.

${ }^{10}$ G.E. Lessings Hamburgische Dramaturgie wird explizit in Ion Barbus Gedicht Balthazar (S. 255f.) erwähnt, das der Dichter dem Literaturkritiker Eugen Lovinescu gewidmet hat. Dort wird G.E. Lessings Ästhetik mit Selbstironie der eigenen Poetik (,Dramaturgia câmpineană“) entgegengestellt, vgl.: „Dar colo-în turnul cel mai trist, / Absurdul liric algebrist / Opune din entelechii / Dramaturgia câmpineană / Hamburgei 
Über die im 18. Jahrhundert historisch bezeugte Hamburger Liebesdienerin Tante Buhr heißt es mit der Selbstironie des Literaten, der sich wie einst G. E. Lessing des großen Themas der antithetischen Beziehung zwischen Natur und Kultur annimmt: „Făptura ei se-nchide-n zinc / Doi ani în urma lui Lessing, / Dar marea faimă a ei de Hură / Din spate, coaste, îl concură!“ (Ebd.)

Überhaupt ist in Ion Barbus Gelegenheitsdichtung die Hafenstadt Hamburg ein mit Pathos beschworener hortus deliciarum - „O, Hamburg! O, clară Hansă!““ (Cu şapte grade ..., S. 253), in dem die erotischen Phantasien des lyrischen Ichs zur Realität werden. In Anspielung auf Casanova nennt sich das erotisch hyperaktive lyrische Ich „Casavechia“ (s. Ispitit..., S. 252), zu dessen ,Eroberungen' auch die weiblich personifizierte Hansestadt Hamburg zählt. In zweideutigen Versen wird die Reise des walachischen Schürzenjägers nach Hamburg durch Verben wie ,a ridica“ und ,,a împlânta“" als Geschlechtsakt grotesk-überdimensionalen Ausmaßes inszeniert: „Ispitit de marea, plina, / Însetata Berolina, / Ridic cortul şi-n amurg / Îl împlânt, hăt, la Hamburg.“ (Ebd.) In der sinnenfreundlichen Hansestadt findet der Mathematiker Dan Barbilian zur Dichtung zurück, was als eine Auferstehung von den Toten gefeiert wird: „Un decedat poet învie / Şi-un Gastprofessor, in Hamburg!"“11 (Iubite Tudor..., S. 261.) Eng verwandt im Geist mit Hamburg ist die Stadt Hannover ein Ort der Freiheit und der erotischen Erfüllung, als deren Perle „Mutter Pisewitt“ dargestellt wird eine historische Studentenkneipe, die Ion Barbu in seiner Jugend eifrig frequentiert hatte: „Nici Amsterdam, nici Haga, nici Düsseldorf renan / Nu au drăgălăşia şi calmul suveran / Al liberei Hanovre, în care am iubit / Atât, şi-a cărei perlă e Mutter Pisewitt.“ (Nici Amsterdam..., S. 279.) Die dionysische Verbindung zwischen Wein und Weib macht aus der Stadt

dramaturgii.“" Das Adjektiv „câmpineană“ ist als Anspielung auf den Namen des Geburtsorts des Dichters zu verstehen, Câmpulung Muscel. ${ }^{11}$ Kursivschrift im Original! 
Hannover einen locus amoenus, der wie ein verlorenes Paradies mit tiefer Nostalgie evoziert wird. Mit derselben Nostalgie evoziert Ion Barbu auch das Berlin seiner Jugend, das Berlin der ,wilden' Zwanziger Jahre: „Ah, unde e Grete şi Fritzi / Şi Agnes cea lungă-n picioare, / Apaşii erotici, bandiţii / Şi multele false fecioare? // Inima-n doliu suspine / După acel Babilon!““ (Oraşul..., S. 251.) Mit Ausnahme Göttingens, der rabenmütterlichen Universitätsstadt, die ihm als dort scheiterndem Doktoranden der Mathematik viel Qual bereitet hat, entbehren die deutschen Metropolen in Ion Barbus Gelegenheitsdichtung jeglicher expressionistischen Dämonie. Die deutschlandbezogene Großstadtlyrik Ion Barbus speist sich eher aus dem sinnenfreudigen, lebensbejahenden Geist der Renaissance und des Humanismus, aus den Trink- und Liebesliedern der Carmina Burana.

In den auf Rumänisch verfassten Gelegenheitsgedichten Ion Barbus begegnet man häufig Entlehnungen aus dem Deutschen, die dem poetischen Diskurs den Charakter einer rein privaten, mit allerlei erotischen Indiskretionen und Pikanterien gespickten Mitteilung, zugleich aber auch Humor und Lokalkolorit verleihen. ${ }^{12}$ Als besonders humorvoll erweist sich Ion Barbus makkaronische Dichtung, in der einer zügellosen Erotik zweisprachig gehuldigt wird. Die spielerische Verschmelzung des Rumänischen mit dem Deutschen gibt im Medium der Sprache die erotische Begegnung des lyrischen Ichs mit deutschen oder Deutsch sprechenden Frauen aus unterschiedlichsten sozialen Schichten wieder, so zum Beispiel mit der bezaubernden

${ }^{12}$ Hier einige weitere Beispiele; Kursivschrift im Original: „La Franciscaner: ceaiul şi-o Abendbrot săracă..." (s. La Franciscaner..., S. 215); „O singură idee în cap: ,Ich bin kaput"“ [sic!] (s. Cu şapca studenţească..., S. 219); „Ochiul mării, fix şi ungeheuer“ (s. Eunuc..., S. 229f.); „Sunt la... bombastul Vaterland!” (s. Cu sacii goi, S. 224f.); „Pe Filmschauspielerin Agnes” (s. ebd.); „Pe al tău immer Casavechia“ (s. Ispitit, S. 252); „Montează-un act de Landungsbrücke“" (s. Balthazar, S. 255f.); "Şi-un Gastprofessor, in Hamburg!“ (s. Iubite Tudor..., S. 261), usw. 
„Schauspielerin Agnes“ (Cu sacii goi..., S. 224f.), mit der in erotischer Ekstase „O, ye“ stöhnenden Else aus Hannover (Vrăjit..., S. 280f.) oder mit einer glühenden Anhängerin Sigmund Freuds, die nach dem Liebesakt verabschiedet wird mit den derb obszönen "makkaronischen” Versen: „Salut şi slavă ţie, o, stusses Wesen, ,Du', / Retorsă Entitate ce astăzi mă futù. “13 (Freud, S. 232.) Unter all diesen Frauen ist Maria, die nicht mehr ganz junge Königin Rumäniens aus dem Hause Sachsen-Coburg und Gotha, zweifelsohne die prominenteste. Das auf Deutsch geführte Gespräch mit der verwitweten, damals nicht mehr regierenden Monarchin in deren Sommerschloss in Sinaia wird im Gedicht La cronica Sinaiei... als unterschwellig erotische Begegnung mit einer alterlosen Schönheitsgöttin besungen:

Căci pentru inimi caste! şi tari, bine născute,

Aphrodité, ca marea, deprimă orice cute;

Că nu există vârstă, declin ori menopaus'

Von einem humanistisch-historischen Punkt aus;

$[\ldots]$

... Sie War [sic!] voll Pracht und Blüte und liebenswürdig und

Bat mich - unwiderstehlich - um einem [sic!] „Joc secund“

Ce destinat îi este demult, de origină.

Deci, am depus volumul în mâna-I de regină... (La cronica Sinaiei..., S. 248f.) $)^{14}$

Der zweideutige Humor der makkaronischen Verse, in denen diese ,Hofberichterstattung' verfasst wurde, gleitet zuweilen ins Sexistische hinüber und kaschiert das satirische Moment des Textes, das man dem Dichter beinahe als Majestätsbeleidigung hätte anlasten können: ,Edictul meu poetic - ştii bine, nu fac caz - / Dar, astăzi, fără voie, el creşte la Ucaz: / Până la culmi

${ }^{13}$ Kursivschrift im Original!

${ }^{14}$ Auch im Falle dieses "makkaronischen" Gedichts, das Rechtschreibfehler und Grammatikfehler enthält, sind Transkriptionsfehler durch den Herausgeber Mircea Coloşenco nicht auszuschließen. 
severe de mare invectivă“ (ebd.). Der Titel des in die Hand der Königin gelegten Lyrikbands verweist im Kontext dieses Gedichts nicht nur auf das Credo Ion Barbus, dass die Poesie als eine höhere Form des Spiels zu verstehen ist, als „un joc secund, mai pur"15, sondern suggeriert zugleich, dass das zweisprachige Gespräch mit der unwiderstehlichen Königin als ein erotisches Spiel zu verstehen ist, ähnlich der spielerischen Vermischung des Rumänischen mit dem Deutschen.

Von besonderem Interesse sind im Rahmen der vorliegenden Untersuchung diejenigen Gelegenheitsgedichte, die Ion Barbu gänzlich auf Deutsch verfasst hat. Diese Gedichte, deren Zahl man an den Fingern einer Hand abzählen kann, vervollständigen das Spektrum der poetischen Experimente, die Ion Barbu hauptsächlich auf Französisch ${ }^{16}$, manchmal auch auf Englisch ${ }^{17}$ gemacht hat. Ihr Duktus beweist, dass Ion Barbu auch auf Deutsch, d.h. in einer Fremdsprache, die er eigenen Aussagen zufolge im Vergleich zu Tudor Vianu eher mäßig beherrschte, seine dichterische Identität dennoch zu artikulieren fähig war. Derselbe Rückgriff aufBilder der antiken Mythologie, den er auch in seiner rumänischen Gelegenheitsdichtung praktiziert hatte, lässt einen poetischen Diskurs entstehen, der um dieselbe obsessive Bejahung der Sexualität kreist: „Du bist der Abfall aller Lichte / Die Unvermeidliche, die Dichte / Der Sünde, Du des Atreus Tiste [sic!] / Du Abgrund dem Geometer lieb.“( $D$ u bist der Abfall..., S. 282.) Der Abgrund der Sexualität entspricht im antiken Mythos um Atreus und Thyestes dem dort

${ }^{15}$ Der Vers entstammt dem berühmten Gedicht Ion Barbus Din ceas, de$d u s . .$. aus dem 1930 veröffentlichten Band Joc secund, s. die im vorliegenden Beitrag verwendete Edition der Werke Ion Barbus, S. 23.

${ }^{16} \mathrm{Zu}$ den auf Französisch verfassten Gelegenheitsgedichten Ion Barbus zählen: Puisque mon âme, S. 211; Pour soulanger..., S. 212; Qu'il fouille, le souvenir..., S. 213; Dan bleu..., S. 222; Mon âme..., S. 236; Héros du potentiel..., S. 239f; Un pur mensonge..., S. 254; Un sage..., S. 264; Le petit Marcel..., S. 298.

${ }^{17}$ Siehe: Sweet Vision, S. 257; The Bridegroom of the Sea, S. 259. 
beschworenen Kannibalismus als einer extremen Manifestationsform des Eros.

Weniger düster klingt das dem Freund Alexandru Rosetti gewidmete Gedicht Meinem besten Alexander ... (S. 276f.), das 1938 in Düsseldorf auf drei Postkarten auf Deutsch geschrieben und dem Freund nach Bukarest geschickt wurde. Das tagebuchartige Gedicht hat insofern Bekenntnis-Charakter, als darin das lyrische Ich dem Freund in der Ferne gesteht, „Eng am Rhein, wo Düssel mündet" (ebd.) Ehebruch nicht nur begangen, sondern auch tief genossen zu haben. Und da der Mensch, so Sigmund Freuds Lehre ${ }^{18}$, der einmal gekannten Lust nicht mehr entbehren möchte, plant das lyrische Ich gleich nach seiner Rückkehr in die walachische Heimat den nächsten Ehebruch: mit keiner anderen Frau als just der Geliebten seines Freundes namens Martha:

$\mathrm{Ja}$, in Neuss schlaf' ich und webe

Träume, die ich hier erlebe,

Schwankend zwischen zwei Kulturen:

Herbe Nonnen, weiche Huren,

Tags in Ly verliebt (Unsinn),

Abends, doch, geb [sic!] ich mich hin

Ganz der jungen Mary Leister

Deren Herr ich bin (und Meister),

Die an mir sehr hängt und oh!

Lockt mich ganz mit ihrem Loch

So dass immer stehe ich hardt [sic!] da

(Wie Du einst bei deiner Martha!)

- Schenkst Du mir sie einmal, gell?

Dein Aufrichtiger

Miguel (Meinem besten Alexander..., S. 276f.) $)^{19}$

${ }^{18}$ Ion Barbu nimmt zwar in seiner Gelegenheitsdichtung die Lehre Sigmund Freuds aufs Korn, s. Freud, S. 232, dennoch folgt er ihr in diesem Gedicht mit der ihm charakteristischen Selbstironie!

${ }^{19}$ Kursivschrift im Original! Im Kommentar zu diesem Gedicht bietet der Herausgeber Mircea Coloşenco eine Übersetzung des Textes ins 
Das im Gedicht Meinem besten Alexander... geschilderte Schwanken zwischen zwei Kulturen bietet sich einer mehrfachen Deutung an: Einerseits handelt es sich dabei um das Schwanken des Dichters zwischen der rumänischen und der deutschen Kultur, andererseits aber um sein Schwanken zwischen Lustprinzip und christlicher Moral, Traum und Wirklichkeit, Augenblick und Ewigkeit, Diesseits und Jenseits.

Die herbei gewünschte Überwindung des antithetischen Verhältnisses zwischen der Welt der rumänischen und der Welt der deutschen Kultur, die Vereinigung beider Kulturen zu einem geistigen europäischen Ganzen, sah Ion Barbu irrtümlicherweise in der nationalsozialistischen Politik des Dritten Reichs sich verwirklichen, einer Politik, deren ,kongeniales' Echo das Programm der rumänischen "Legionärsbewegung" um Corneliu Zelea Codreanu war. Der exaltierte Panegyrikus, 1940 betitelt, den der Dichter - inzwischen zum prominenten Mitglied der Legionärsbewegung avanciert - dem Führer Adolf Hitler und dem Haupt des rumänischen Faschismus Corneliu Zelea Codreanu gewidmet hat, zeigt in erschütternder Art und Weise, wie Ion Barbus Liebe und Bewunderung für Deutschland, ein Land, das er sein großes, zweites Heimatland nannte - „Dar dreptul Vlah [...] strânge-n el icoana / Acestei mari, a doua ţări a lui“" (1940, S. 187) -, in politische Verblendung umgeschlagen

Rumänische durch Fred Popovici, die er der Übertragung durch Ilinca Iuraşcu wegen ihrer angeblich größeren Textnähe vorzieht, s. S. 783f. Die Übersetzung Fred Popovicis ist jedoch voll von groben Verständnisfehlern, z. B.: „Von dem [sic!] Wissenschaftler's Los / Gott sei Dank, jetzt bin ich los!“ wurde sinnentstellend übersetzt durch: „De a învătatului treabă, / Slavă Domnului, acum mă ocup!““(S. 783); „Herbe Huren“" wurde sinnentstellend übersetzt durch „Călugăriţe fleşcăite“ (ebd.); der Vers: „Die [...] lockt mich ganz mit ihrem Loch“ wurde fälschlicherweise übersetzt durch: „Şi mă încuie cu gura-i îmbietoare“ (S. 784). Diese von der Rumänischen Akademie preisgekrönte Edition des poetischen Werks Ion Barbus aus dem Jahr 2000 bedarf einer tiefgreifenden Revision der deutschsprachigen Texte des Dichters und deren Übersetzung ins Rumänische! 
war. In der Gestalt des Führers sieht Ion Barbu in diesem Loblied das Genie der deutschen Kultur verkörpert, das sich im Laufe der Jahrhunderte durch Dichter wie Novalis und durch Wissenschaftler von Weltrang wie den Mathematiker Bernhard Riemann, den bärtigen Gott Göttingens, ${ }^{20}$ manifestiert hatte. Ion Barbu verklärt Deutschland zum rettenden Ufer des rumänischen Geistes, den der Liberalismus der 1848er Revolution angeblich verraten und vernichtet hatte. In der Gestalt des Führers Adolf Hitler und des Kapitäns Corneliu Zelea Codreanu sieht er Helden, welche von der Vorsehung mit einer heilbringenden Mission betraut worden waren, nämlich Europa zu einer übernationalen geistigen Einheit im Zeichen des Hakenkreuzes zu formen und sich die ganze Welt zu Füßen zu legen:

Noi nu uităm, că duhului ce-învinge,

În veacul vlah, uscat ai fost şi istm,

Că ,iarba de omăt”, a noastră, întinge

În despletitul tău idealism;

Iar cea mai pură creştere din toate,

El, începutul, cel ce ne e tot:

Prea sfântul Căpitan, cuprinde poate

În strat adânc şi osemântul got.

Deci, slavă, mântuire, Fuehrer, ţie!

- Legiunea trage verdele-i covor,

Să treci în mers, de altă Alexandrie,

La Darius şi Împăratul Por.

Die politischen Irrwege, die Ion Barbu in den 40er Jahren gegangen ist, überschatteten lange Zeit seine literarischen Verdienste. In der rumänischen Literaturgeschichtsschreibung hat man bis heute nicht vergessen, dass Ion Barbu als weltweit berühmter Mathematiker sich ostentativ im grünen Hemd dem Symbol der faschistischen "Legionärsbewegung" - in der Bukarester Universität erblicken ließ. Seinen nicht weniger berühmten Zeitgenossen, dem Philosophen Emil Cioran und dem

${ }^{20}$ Siehe: „Riemann, zeul tău bărbos“, 1940, S. 188. 
Religionshistoriker Mircea Eliade, die aus ihrer Begeisterung für die faschistische "Legionärsbewegung" keinen Hehl gemacht haben, hat man diese Irrungen und Wirrungen - um mit Theodor Fontane zu sprechen - leichter verziehen. Ion Barbu hingegen, der Deutschland sein zweites, großes Heimatland nannte, hat für seine zweifelhaften politischen Sympathien mit einem langjährigen Ausschluss aus der rumänischen Literaturgeschichte büßen müssen. Heute darf er sich wieder zu den Klassikern der rumänischen Moderne zählen. Modern ist Ion Barbus Dichtung nicht zuletzt auch dank ihres ausgeprägten interkulturellen Moments.

\section{Bibliographie}

Barbu, Ion: Opere. I. Versuri. Ediţie alcătuită de Mircea Coloşenco. Bucureşti, 2000.

Heine, Heinrich: Sämtliche Schriften in zwölf Bänden. Band 1. Schriften 1817-1840. Herausgegeben von Klaus Briegleb, Frankfurt am Main, Berlin, Wien, 1981. 